\title{
Association Between In-Clinic Opioid Administration and Discharge Opioid Prescription in Urgent Care: a Retrospective Cohort Study
}

\author{
Susan L. Calcaterra, MD, MPH ${ }^{\top}$ (1), Yingbo Lou, $M S^{2}$, Rachel M. Everhart, $P h D, M S^{1,2}$, \\ Lindsey E. Fish, $M D^{1,2}$, and Rebecca Hanratty, $M D^{1,2}$
}

'Division of General Internal Medicine, Department of Medicine, University of Colorado Aurora, CO, USA; ${ }^{2}$ Ambulatory Care Services, Denver Health Medical CenterDenver, CO, USA.

BACKGROUND: Emergency departments increasingly use nonopioid analgesics to manage acute pain and minimize opioid-related harms. Urgent care centers are expanding to lower costs and provide efficient access to healthcare. General internists increasingly work in these acute care settings. Much is known about opioid prescribing in the primary care, inpatient, and emergency department setting. Little is known about opioid prescribing in the urgent care setting and associated outcomes.

OBJECTIVES: To assess the association between in-clinic opioid administration and opioid receipt at clinic discharge and on progression to chronic opioid use among urgent care patients.

DESIGN: Retrospective cohort study.

PARTICIPANTS: Patients, 20 years or older and not on opioid medications, who presented for care to an urgent care clinic within a safety-net healthcare system from June 1, 2016, to April 30, 2019.

MAIN MEASURES: We examined the association between the in-clinic administration of oral or intravenous opioids and opioid receipt at clinic discharge. We also examined the association between in-clinic opioid administration and progression to chronic opioid use after six months.

KEY RESULTS: The study sample included 34,978 patients, of which $13.8 \%(n=4842)$ received in-clinic opioids and $86.2 \%(n=30,136)$ did not receive in-clinic opioids. After adjusting for age, gender, race/ethnicity, insurance, and pain diagnosis, patients who received in-clinic opioids were more likely to receive opioids at discharge compared to patients who did not receive in-clinic opioids $(\mathrm{aOR}=12.30,95 \%$ CI 11.44-13.23). Among a selected cohort of patients, in-clinic opioid administration was associated with progression to chronic opioid use $(\mathrm{aOR}=$ 2.12, 95\% CI 1.66-2.71).

CONCLUSIONS: In-clinic opioid administration was strongly associated with opioid receipt at discharge and progression to chronic opioid use. Increased use of nonopioid analgesics in urgent care could likely reduce this association and limit opioids available for diversion, overdose, and death.

Electronic supplementary material The online version of this article (https://doi.org/10.1007/s11606-020-06059-8) contains supplementary material which is available to authorized users.

Received March 25, 2020

Accepted July 13, 2020

Published online July 29, 2020
KEY WORDS: urgent care; opioids; acute pain.

J Gen Intern Med 36(1):43-50

DOI: $10.1007 / \mathrm{s} 11606-020-06059-8$

(c) Society of General Internal Medicine 2020

\section{BACKGROUND}

Opioids are an effective treatment for pain management in the acute care setting and are beneficial to reduce pain and suffering. Unfortunately, opioid prescribing has associated harms, including progression to chronic opioid use, ${ }^{1}$ development of opioid use disorder, ${ }^{2}$ and risk of opioid diversion and overdose. ${ }^{3}$ Increasingly, alternatives to opioid medications for acute pain management are used as a first-line approach in emergency departments (ED) and inpatient hospital settings. This includes the use of nonopioid analgesic to manage commonly encountered painful diagnoses such as musculoskeletal pain, low back pain, migraine or other headache pain, extremity fracture or joint dislocation, and renal colic. ${ }^{4,5}$ Early studies demonstrate a decrease in overall opioid use without a change in patient satisfaction for pain control ${ }^{5}$ suggesting that the use of nonopioid alternatives is a feasible and reasonable first-line approach for pain management.

Healthcare systems increasingly use urgent care centers to provide acute care, to lower costs, and to provide efficient access to healthcare. ${ }^{6,7}$ The expansion of urgent care clinics has led to an increase in internal medicine clinicians working in the urgent care settings. ${ }^{8}$ While much attention has focused on opioid prescribing in primary care ${ }^{9}$ and hospital settings, ${ }^{10}$ little is known about opioid prescribing practices in urgent care.

This investigation aimed to identify opioid prescribing practices in two urgent care clinics predominantly staffed by internal medicine and family medicine clinicians. We present the results of a natural experiment which evolved from the opening of a second urgent care clinic affiliated with a safetynet healthcare system in Denver, Colorado. In this new urgent care clinic, hospital administrators opted out of on-site storage for DEA scheduled II and III controlled medications. ${ }^{11}$ Thus, patients cared for at this urgent care setting were managed with nonopioid analgesics or tramadol for acutely painful 
conditions. The original urgent care clinic had access to opioids on-site. This study examines a cohort of patients with acutely painful conditions not on opioid therapy who presented to urgent care. We assessed the association between inclinic opioid administration and opioid receipt at discharge and on progression to chronic opioid use among urgent care patients.

\section{METHODS}

\section{Study Design and Setting}

This was a retrospective cohort study of patients who received care at either of two urgent care clinics within a large academic safety-net healthcare system, Denver Health, from June 1, 2016, to April 30, 2019. One urgent care clinic is adjacent to an urban emergency department located in a county hospital (ED-based urgent care). The other urgent care clinic is adjacent to a family health center (free-standing urgent care). This study was determined to be exempt by the Colorado Multiple Institution Review Board.

\section{Data Source and Participants}

We examined all patient encounters to two urgent care clinics via a query of electronic health records. Pharmacy data queried included clinically administered opioid medications at the index encounter reported as intravenous or oral (count) and milligram of morphine equivalents (MMEs) administered. Opioids prescribed at the index encounter discharge were reported as the quantity of tabs prescribed, the total days' supply per prescription, and the total number of prescriptions. Opioids were identified using National Drug codes (NDC) ${ }^{12}$ for codeine, fentanyl, hydrocodone, methadone, morphine, oxycodone, oxymorphone, tramadol, and hydromorphone. We excluded buprenorphine.

We identified all patient encounters to the ED-based urgent care or the free-standing urgent care during the study period. From these encounters, we limited our sample to include patients $\geq 20$ years old and with a chief complaint or discharge diagnoses including acute or chronic radicular back pain, musculoskeletal pain, migraines/recurrent primary headache, extremity fracture or joint dislocation, gastroparesis-associated or chronic functional abdominal pain, or renal colic (Appendix 1). These diagnoses were chosen due to prior studies demonstrating efficacy in pain control using nonopioid alternative medications. ${ }^{4,5}$ Of these encounters, we identified the first encounter to the urgent care clinic during the study period and designated that encounter as the "index encounter." We then excluded patients who filled an opioid prescription at any Denver Health-affiliated pharmacy in the three months preceding their index encounter. Our final sample included patients $\geq 20$ years old without opioid use in the previous 3 months with acute pain.

\section{Exposure Variable}

Patients who received in-clinic intravenous or oral opioids during their index encounter were categorized into the "inclinic opioid" cohort. Patients who did not receive in-clinic intravenous or oral opioids at their index encounter were categorized into the "no in-clinic opioid" cohort.

\section{Outcomes Variables}

The primary outcome measure was receipt (yes/no) of an opioid prescription at the index urgent care discharge.

Our secondary outcome measure described progression to chronic opioid use, defined as a "90-day or greater supply of non-parenteral opioids with less than a 30-day gap in supply within a 180-day period. ${ }^{\prime 13}$ For this outcome, we identified opioid prescriptions filled at a Denver Health-affiliated pharmacy over 6 months following the index encounter. To more accurately identify progression to chronic opioid use, we limited our sample to "empaneled patients," defined as patients who accessed primary care at Denver Health in the 18 months prior to their index encounter.

\section{Baseline Measures}

Gender, race/ethnicity, age, and insurance status were determined from registration data collected at the index encounter. Insurance status was classified as Medicaid, Child Health Plan Plus, Medicare, uninsured/unknown/other/self-pay, commercial, or the system's discount payment plan (Colorado Indigent Care Program; Denver Health Financial Assistance Program). Pain diagnoses at the index encounter were classified per the categories above. Substance use disorder diagnoses were identified by querying patient encounters 1 year following the index encounter using ICD-10-CM codes (Table 1). We calculated a Charlson Comorbidity Index score ${ }^{14}$ using discharge diagnoses from or within 1 year of the index encounter (Table 1).

\section{Primary Data Analysis}

The "in-clinic opioid" and "no in-clinic opioid" cohorts were compared across baseline measures. We used the chi-square test for proportions and Student's $t$ test for continuous variables for unadjusted analyses. We estimated the likelihood of receipt of an opioid prescription at discharge following inclinic opioid administration during the index visit using a multivariate logistic regression model. We controlled for age at index encounter, gender, race/ethnicity, insurance, and pain diagnosis. Among empaneled patients, a second multivariate logistic regression model was used to estimate progression to chronic opioid use following in-clinic opioid administration during the index encounter. This model controlled for age at 
Table 1 Encounter/Patient Demographics $(n=34,978)$

\begin{tabular}{|c|c|c|c|}
\hline & $\begin{array}{l}\text { In-clinic opioid } \\
(n=4842)\end{array}$ & $\begin{array}{l}\text { No in-clinic opioid } \\
(n=30,136)\end{array}$ & $P$ value \\
\hline Age in years (mean, SD) & $41.1(13.8)$ & $40.5(14.6)$ & \multirow{6}{*}{$\begin{array}{l}0.006 \\
<0.001\end{array}$} \\
\hline \multicolumn{3}{|l|}{ Age in groups $(n, \%)$} & \\
\hline $20-25$ & $630(13.0)$ & $4762(15.8)$ & \\
\hline $26-40$ & $1939(40.0)$ & $12,179(40.4)$ & \\
\hline $41-64$ & $1983(41.0)$ & $11,164(37.1)$ & \\
\hline $65+$ & $290(6.0)$ & $2031(6.7)$ & \\
\hline Gender (male) & $2253(46.5)$ & $14,670(48.7)$ & 0.005 \\
\hline \multicolumn{3}{|l|}{ Race $(n, \%)$} & \multirow[t]{5}{*}{0.20} \\
\hline White & $3593(74.2)$ & $22,490(74.6)$ & \\
\hline African American & $613(12.7)$ & $3698(12.3)$ & \\
\hline Asian/Pacific Islander & $100(2.1)$ & $752(2.5)$ & \\
\hline Other/unknown & $536(11.0)$ & $3196(10.6)$ & \\
\hline \multicolumn{3}{|l|}{ Ethnicity $(n, \%)$} & \multirow[t]{4}{*}{$<0.001$} \\
\hline NHW & $2661(55.0)$ & $15,553(51.6)$ & \\
\hline Hispanic & $2151(44.4)$ & $14,195(47.1)$ & \\
\hline Unknown & $30(0.6)$ & $388(1.3)$ & \\
\hline \multicolumn{3}{|l|}{ Clinic location } & \multirow{3}{*}{$<0.001$} \\
\hline Free-standing urgent care $(n=8702)$ & $371(7.7)$ & $8331(27.6)$ & \\
\hline ED-based urgent care $(n=26,276)$ & $4471(92.3)$ & $21,805(72.4)$ & \\
\hline \multicolumn{3}{|l|}{ Primary insurance $(n, \%)$} & \multirow[t]{6}{*}{0.002} \\
\hline Medicaid/CHP & $2289(47.3)$ & $13,725(45.6)$ & \\
\hline Medicare & $501(10.4)$ & $2822(9.4)$ & \\
\hline Uninsured/self-pay/other/unknown & $887(18.3)$ & $5648(18.7)$ & \\
\hline Commercial & $757(15.6)$ & $5316(17.6)$ & \\
\hline CICP/DFAP & $408(8.4)$ & $2625(8.7)$ & \\
\hline \multicolumn{3}{|c|}{ Chief complaint or discharge diagnosis (see Table 3 for associated ICD 10 codes below) } & \multirow{7}{*}{$<0.001$} \\
\hline Acute on chronic radicular back pain & $795(16.4)$ & $4471(14.8)$ & \\
\hline MSK pain & $1170(24.2)$ & $9722(32.3)$ & \\
\hline Migraine/recurrent primary headache & $190(3.9)$ & $2703(9.0)$ & \\
\hline Extremity fracture or joint dislocation & $1529(31.6)$ & $9721(32.3)$ & \\
\hline Gastroparesis-associated or chronic functional abdominal pain & $874(18.1)$ & $3240(10.8)$ & \\
\hline Renal colic & $284(5.9)$ & $279(0.9)$ & \\
\hline \multicolumn{4}{|l|}{ Substance use disorders in ICD $10(n, \%)^{*}$} \\
\hline Alcohol-related disorders & $501(10.4)$ & $2573(8.5)$ & $<0.001$ \\
\hline Opioid-related disorders & $181(3.7)$ & $785(2.6)$ & $<0.001$ \\
\hline Cannabis-related disorders & $201(4.2)$ & $909(3.0)$ & $<0.001$ \\
\hline Sedative-, hypnotic-, or anxiolytic-related disorders & $14(0.3)$ & $83(0.3)$ & 0.87 \\
\hline Cocaine-related disorders & $100(2.1)$ & $496(1.7)$ & 0.03 \\
\hline Other stimulant-related disorders & $143(3.0)$ & $802(2.7)$ & 0.24 \\
\hline Nicotine dependence/use disorder & $848(17.5)$ & $3690(12.2)$ & $<0.001$ \\
\hline Charlson Comorbidity Index (mean, SD) & $0.38(1.1)$ & $0.29(0.96)$ & $<0.001$ \\
\hline \multicolumn{4}{|l|}{ Subsequent encounters (within 90 days) (mean, SD) } \\
\hline Urgent care encounter & $1.34(0.71)$ & $1.41(0.77)$ & 0.06 \\
\hline Emergency room encounter & $1.75(1.11)$ & $1.71(1.37)$ & 0.61 \\
\hline Primary care encounter & $2.74(2.58)$ & $2.53(2.29)$ & $<0.001$ \\
\hline Specialty encounter & $2.19(1.49)$ & $1.95(1.43)$ & $<0.001$ \\
\hline Hospital admission & $5.01(4.76)$ & $5.00(6.43)$ & 0.99 \\
\hline IV opioids administered during index encounter & $1243(25.7)$ & N/A & \\
\hline MMEs administered for IV opioids per person/per encounter? (mean, SD) & $9.3(5.1)$ & N/A & \\
\hline Oral opioids administered during index encounter & $3782(78.1)$ & N/A & \\
\hline MMEs administered for oral opioids per person/per encounter? (mean, SD) & $6.8(3.1)$ & $\mathrm{N} / \mathrm{A}$ & \\
\hline Quantity of tabs per opioid prescription (mean, SD) $(n=5106)$ & $16.5(11.7)$ & $18.6(19.5)$ & $<0.001$ \\
\hline Total days' supply per opioid prescription & $3.7(2.0)$ & $3.9(2.5)$ & 0.002 \\
\hline Number of opioid prescriptions & $2698(55.7)$ & $2744(9.1)$ & $<0.001$ \\
\hline Opioids filled at a Denver Health pharmacy within 12 months of index visi & 64) & & \\
\hline 0 & $763(98.8)$ & $881(98.8)$ & 1.00 \\
\hline $1-2$ & $9(1.2)$ & $10(1.1)$ & \\
\hline $3-4$ & 0 & $1(0.1)$ & \\
\hline$\geq 5$ & 0 & 0 & \\
\hline Opioids filled at a Denver Health pharmacy within 90 days of index encour & J) $(n=1663)$ & & \\
\hline & $772(28.6)$ & $891(32.5)$ & 0.002 \\
\hline $\mathrm{N}$ & 1926 (71.4) & $1853(67.5)$ & \\
\hline Chronic opioid use among empaneled patients ( 6 months post index dischar & N), $n(\%)$ & & \\
\hline Y & $93(4.0)$ & $285(1.9)$ & $<0.001$ \\
\hline $\mathrm{N}$ & $2234(96.0)$ & $14,733(98.1)$ & \\
\hline
\end{tabular}

*Substance use disorder diagnoses and ICD 10-CM code: alcohol-related disorders (F10.XX); opioid-related disorders (F11.XX); cannabis-related disorders (F12.XX); sedative-, hypnotic-, or anxiolytic-related disorders (F13.XX); cocaine-related disorders (F14.XX); other stimulant-related, amphetamine, and methamphetamine disorders (F15.XX); nicotine dependence/use disorder (F17.XX)

index encounter, gender, race/ethnicity, insurance, and pain diagnosis.
Model fit was assessed using the deviance and Pearson goodness of fit tests and the concordance statistic. All analyses 
were conducted using SAS Enterprise Guide version 7.11 HF3 (SAS Institute).

\section{RESULTS}

\section{Characteristics of Study Subjects}

From June 1, 2016, to April 30, 2019, there were a total of 170,580 urgent care encounters. From these, encounters without a pain-related diagnosis $(n=96,706)$ or age $<20$ years old $(n=$ $25,497)$ were excluded. Next, encounters were deduplicated to only include the index encounter $(n=12,819)$. Patients were further excluded if they filled an opioid prescription within the 3 months preceding their index encounter $(n=580)$. Our remaining sample included 34,978 eligible patients. Of these, $13.8 \%(n=4842)$ had in-clinic opioid administration and $86.2 \%(n=30,136)$ did not have in-clinic opioid administration at their index encounter (Fig. 1).

Patients with in-clinic opioid administration were slightly older (mean in years, 41.1, standard deviation [SD] 13.8 versus 40.5 , SD $14.6 p<0.006)$, were more likely to be non-Hispanic White (55.0\% versus $51.6 \%)$, and were more likely to have abdominal pain ( $18.1 \%$ versus $10.8 \%)$ and renal colic $(5.9 \%$ versus $0.9 \%)$ compared to patients without in-clinic opioid administration. In-clinic opioid recipients were more likely to have a diagnosis of alcohol-related disorders $(10.4 \%$ versus $8.5 \%, p<0.001)$, opioid-related disorders $(3.7 \%$ versus $2.6 \%, p<0.001)$, cannabis-related disorders $(4.2 \%$ versus $3.0 \%, p<0.001)$, and nicotine dependence/use disorders (17.5\% versus $12.2 \%$, $p<0.001)$ compared to patients without in-clinic opioid receipt. Patients who received in-clinic opioids had a higher burden of medical illness (Charlson Comorbidity Index of 0.38 versus $0.29, p<0.001$ ) than patients who did not receive in-clinic opioids (Table 1).

Of the 4842 patients with in-clinic opioid receipt, $25.7 \%$ $(n=1243)$ received intravenous opioids with a mean of 9.3 MMEs (standard deviation [SD] 5.1). Oral opioids were administered to $78.1 \%(n=3782)$ of patients with a mean dose of 6.8 MMEs (SD 3.1). At the free-standing urgent care clinic, tramadol was the most common in-clinic opioid administered (98.6\%, $n=362)$ followed by codeine/guaifenesin $(1.4 \%, n=$ $5)$. At the ED-based urgent care clinic, hydrocodoneacetaminophen was the most common in-clinic opioid administered $(33.0 \%, n=1633)$ followed by oxycodoneacetaminophen $(n=1147,23.2 \%)$ and intravenous morphine $(21.6 \%, n=1069)$ (data not shown). Among the study sample, there were 5442 opioid prescriptions ordered at discharge. Of the patients who received in-clinic opioids, $55.7 \%(n=2698)$ were prescribed opioids at discharge. Of the patients who did not receive in-clinic opioids, $9.1 \%(n=2744)$ were prescribed opioids at discharge $(p<0.001)$ (Table 1$)$.

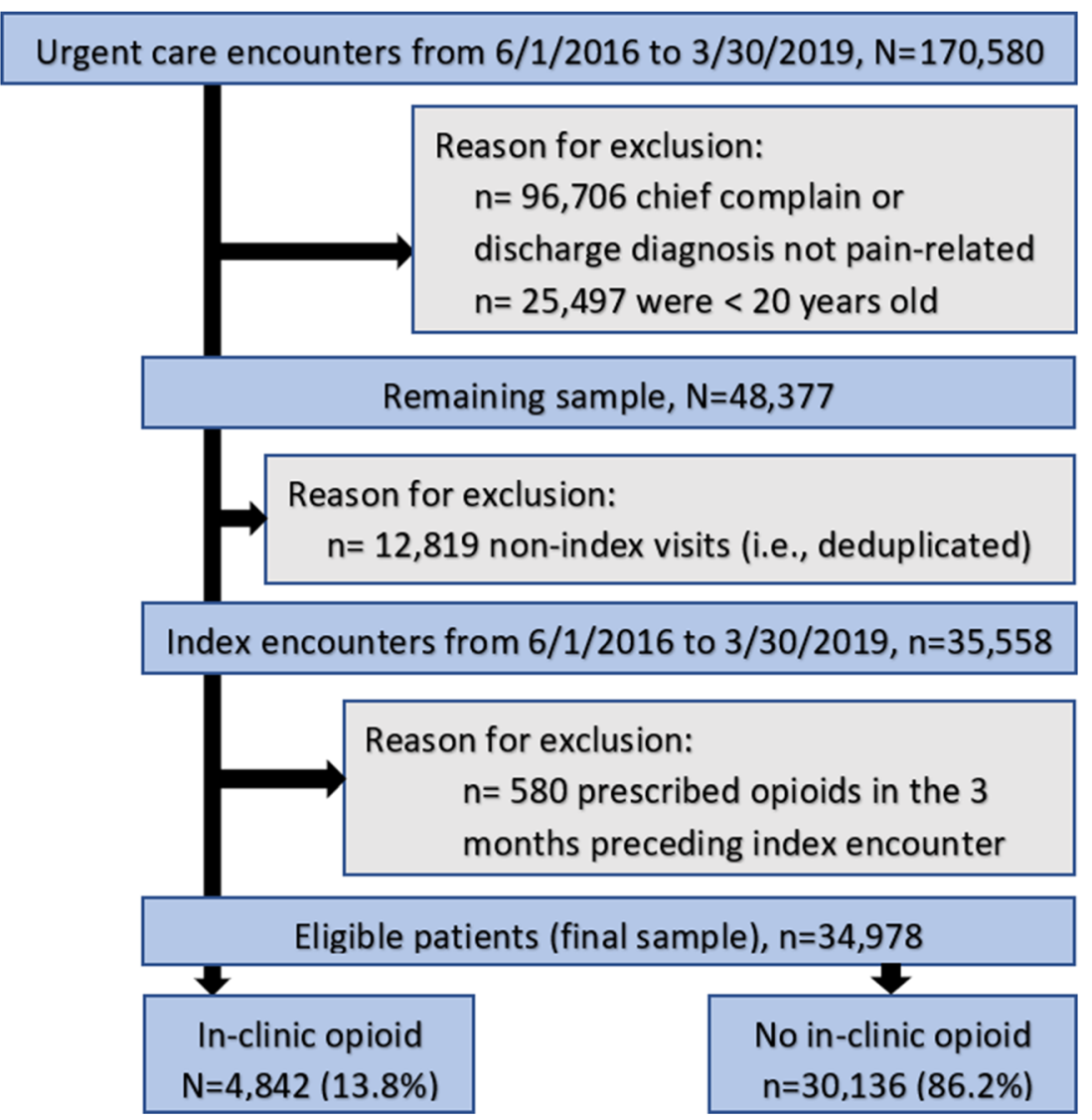

Figure 1 Patient flow chart. 
There were 1664 opioid prescriptions filled at an affiliated pharmacy within 90 days of the index encounter. Of these opioid prescriptions, $46.4 \%(n=772)$ were filled by patients who received in-clinic opioids and $53.6 \%(n=891)$ were filled by patients who did not receive in-clinic opioids $(p<0.001)$. Among empaneled patients $(n=17,345)$, in-clinic opioid recipients were more likely to progress to chronic opioid use after 6 months compared to patients who did not receive inclinic opioids ( $4 \%$ vs. $1.9 \%, p<0.001$ ) (Table 1 ).

\section{Primary Outcome Data: Receipt of an Opioid Prescription Within $72 \mathrm{~h}$ of Discharge}

Patients with in-clinic opioid receipt were more likely to be prescribed opioids at discharge compared to patients without in-clinic opioid receipt (adjusted odds ratio $[\mathrm{aOR}]=12.30$, 95\% Confidence Interval [CI] 11.44-13.23). Patients with fractures or joint dislocations $(\mathrm{aOR}=1.46,95 \%$ CI 1.32 1.61) or renal colic $(\mathrm{aOR}=8.60,95 \%$ CI 6.89-10.74) were more likely to receive an opioid prescription at discharge compared to patients with acute or chronic radicular back pain. Older patients were more likely to receive an opioid prescription at discharge $(\mathrm{aOR}=1.01,95 \%$ CI 1.01-1.01). Patients with gastroparesis $(\mathrm{aOR}=0.62,95 \% \mathrm{CI} 0.54-0.71)$ or migraines (aOR $0.37,95 \%$ CI $0.30-0.45)$ were less likely to receive an opioid prescription at discharge compared to patients with acute or chronic radicular back pain (Table 2).

A secondary analysis limited to patients seen at the EDbased urgent care demonstrated a slightly lower association between in-clinic opioid receipt and prescribed opioids at discharge $(\mathrm{aOR}=12.0,95 \%$ CI 11.1-13.0).

\section{Secondary Outcome Data: Progression to Chronic Opioid Use Among Empaneled Patients}

When limiting the analysis to empaneled patients, those who progressed to chronic opioid use within 6 months following their index encounter were more likely to have in-clinic opioid receipt during their index encounter $(\mathrm{aOR}=2.12,95 \% \mathrm{CI}$ 1.66-2.71). Patients with gastroparesis $(\mathrm{aOR}=0.49,95 \% \mathrm{CI}$ $0.29-0.83$ ) or migraines $(\mathrm{aOR}=0.46,95 \%$ CI $0.25-0.84$ ) were less likely progress to chronic opioid use compared to patients with acute or chronic radicular back pain. Older age $(\mathrm{aOR}=1.03,95 \%$ CI $1.02-1.04)$ and male $(\mathrm{aOR}=1.23,95 \%$ CI 1.02-1.55) patients were both more likely to proceed to chronic opioid use. Patients with Medicaid $(\mathrm{aOR}=1.69,95 \%$ CI 1.10-2.60) and Medicare ( $\mathrm{aOR}=2.57,95 \%$ CI 1.59-4.15) were more likely to progress to chronic opioid use compared to patients with commercial insurance (Table 3).

\section{DISCUSSION}

In this retrospective cohort study of patients presenting to urgent care for painful diagnoses, patients who received inclinic opioids had more than tenfold odds of receiving opioids at discharge compared to patients who did not receive in-clinic opioids, even after controlling for presenting diagnoses. Similarly, among a cohort of empaneled patients, those who received in-clinic opioids had twice the odds of progressing to chronic opioid use after 6 months compared to patients who did not receive in-clinic opioids. These findings extend the

Table 2 Adjusted Odds Ratio for Receipt of an Opioid at Discharge Among All Index Encounters Effect $(n=34,978)$

\begin{tabular}{|c|c|c|c|c|}
\hline & Odds ratio estimate & \multicolumn{2}{|c|}{$\begin{array}{l}\text { Odds ratio (95\% } \\
\text { confidence limits) }\end{array}$} & $P$ value \\
\hline \multicolumn{5}{|l|}{ In-clinic opioid administration } \\
\hline No (ref) & - & - & - & - \\
\hline Yes & 12.30 & 11.44 & 13.23 & $<0.0001$ \\
\hline \multicolumn{5}{|l|}{ Chief complaint or discharge diagnosis } \\
\hline Chronic radicular back pain (baseline) & - & - & - & - \\
\hline Extremity fracture or joint dislocation & 1.46 & 1.32 & 1.61 & $<0.0001$ \\
\hline Gastroparesis-associated or chronic functional abdominal pain & 0.62 & 0.54 & 0.71 & $<0.0001$ \\
\hline Musculoskeletal pain & 0.92 & 0.83 & 1.02 & 0.13 \\
\hline Migraine/recurrent primary headache & 0.37 & 0.30 & 0.45 & $<0.0001$ \\
\hline Renal colic & 8.60 & 6.89 & 10.74 & $<0.0001$ \\
\hline Age & 1.01 & 1.01 & 1.01 & $<0.0001$ \\
\hline \multicolumn{5}{|l|}{ Gender } \\
\hline F (ref) & - & - & - & - \\
\hline $\mathrm{M}$ & 1.0 & 0.93 & 1.07 & 0.88 \\
\hline \multicolumn{5}{|l|}{ Race/ethnicity } \\
\hline White (ref) & & - & - & - \\
\hline African American & 0.93 & 0.83 & 1.04 & 0.18 \\
\hline Asian & 0.91 & 0.72 & 1.15 & 0.44 \\
\hline Hispanic & 1.04 & 0.97 & 1.13 & 0.29 \\
\hline Other/unknown & 0.86 & 0.68 & 1.09 & 0.21 \\
\hline \multicolumn{5}{|l|}{ Insurance } \\
\hline Commercial (ref) & & - & - & - \\
\hline Financial assistance & 0.83 & 0.71 & 0.96 & 0.01 \\
\hline Medicaid & 0.99 & 0.90 & 1.09 & 0.79 \\
\hline Medicare & 0.84 & 0.73 & 0.98 & 0.03 \\
\hline Uninsured/other/unknown & 0.89 & 0.79 & 0.10 & 0.04 \\
\hline
\end{tabular}


Table 3 Adjusted Odds Ratio for Progression to Chronic Opioid Use Within 6 Months of Index Discharge Among Empaneled Patients $(n=$ 17,101)

\begin{tabular}{|c|c|c|c|c|}
\hline & Odds ratio estimate & \multicolumn{2}{|c|}{$\begin{array}{l}\text { Odds ratio (95\% } \\
\text { confidence limits) }\end{array}$} & $P$ value \\
\hline \multicolumn{5}{|l|}{ In-clinic opioid administration } \\
\hline No (ref) & - & - & - & - \\
\hline Yes & 2.12 & 1.66 & 2.71 & $<0.0001$ \\
\hline \multicolumn{5}{|l|}{ Chief complaint or discharge diagnosis } \\
\hline Chronic radicular back pain (baseline) & & - & - & - \\
\hline Extremity fracture or joint dislocation & 0.84 & 0.62 & 1.14 & 0.26 \\
\hline Gastroparesis-associated or chronic functional abdominal pain & 0.49 & 0.29 & 0.83 & 0.01 \\
\hline Musculoskeletal pain & 0.93 & 0.70 & 1.22 & 0.59 \\
\hline Migraine/recurrent primary headache & 0.46 & 0.25 & 0.84 & 0.01 \\
\hline Renal colic & 0.38 & 0.09 & 1.56 & 0.18 \\
\hline Age & 1.03 & 1.02 & 1.04 & $<0.0001$ \\
\hline \multicolumn{5}{|l|}{ Gender } \\
\hline F (ref) & - & - & - & - \\
\hline $\mathrm{M}$ & 1.26 & 1.02 & 1.55 & 0.03 \\
\hline \multicolumn{5}{|l|}{ Race/ethnicity } \\
\hline White (ref) & - & - & - & - \\
\hline African American & 0.96 & 0.69 & 1.34 & 0.81 \\
\hline Asian & 0.10 & 0.01 & 0.75 & 0.02 \\
\hline Hispanic & 1.18 & 0.93 & 1.51 & 0.17 \\
\hline Other/unknown & 1.54 & 0.77 & 3.10 & 0.22 \\
\hline \multicolumn{5}{|l|}{ Insurance } \\
\hline Commercial (ref) & - & - & - & - \\
\hline Financial assistance & 0.50 & 0.26 & 0.96 & 0.04 \\
\hline Medicaid & 1.692 & 1.10 & 2.60 & 0.02 \\
\hline Medicare & 2.57 & 1.59 & 4.15 & 0.00 \\
\hline Uninsured/other/unknown & 0.24 & 0.07 & 0.80 & 0.02 \\
\hline
\end{tabular}

current literature which demonstrate an association between initial opioid prescription receipt and progression to chronic opioid use in the $\mathrm{ED}^{2}$ and hospital setting ${ }^{15}$ to include the urgent care setting.

Urgent care facilities offer a less-costly alternative to the ED for healthcare access. ${ }^{16}$ Many of the pain-related conditions treated in EDs are also treated in urgent care settings. ${ }^{17}$ Urgent care services continue to grow to meet the needs of the healthcare consumer. ${ }^{7}$ Given past experience with opioid prescribing in the ED, and the increasing trend to manage pain with nonopioid analgesics, it is important to examine pain management practices in urgent care. This is especially important given the rapid growth of urgent care centers, where opioid prescribing may greatly contribute to increased opioid use, both acutely and chronically. Previous research has demonstrated an association between opioid prescribing and the availability of opioids for diversion, ${ }^{18}$ leading to an increase in opioid overdose deaths. ${ }^{19}$ To mitigate these outcomes, EDs are increasingly using alternatives to opioids to treat painful conditions. ${ }^{20-22}$ If and how urgent care centers are implementing similar pain management strategies should be examined. There may be an opportunity to reduce opioid prescribing in urgent care settings and to expand the use of nonopioid analgesics for pain management.

There are times when opioid administration is necessary and effective. We found that patients with an extremity fracture or joint location were more likely to receive an opioid prescription at discharge. We do not suggest that opioids should not be used when a patient's pain is severe and uncontrolled. Furthermore, we do not attempt to discern the appropriateness of in-clinic opioid administration, opioid prescribing at discharge, or progression to chronic opioid use. This study aimed to investigate the association between inclinic opioid administration, opioid receipt at discharge, and progression to chronic opioid use. Recognizing that opioids may be the best medication available to manage an acutely painful process, and that the free-standing urgent care did not have DEA scheduled II and III opioids available, we reviewed patient transfers from the free-standing urgent care clinic to the ED. This review revealed very few transfers for uncontrolled pain (data not shown) suggesting that the effectiveness of nonopioid analgesic alternatives for acutely painful conditions is a viable option for pain management in the urgent care setting.

This study occurred in an integrated, safety-net healthcare system with two urgent care clinics. Physicians employed at these clinics are affiliated with an academic institution. The patient population was mostly White, included both non-Hispanic and Hispanic patients, and many patients were insured with state Medicaid. These findings may not be generalizable to for-profit urgent care clinics where patients pay out-of-pocket for services. These results may not be generalizable to retail walk-in clinics or "minute clinics" where the care provided is limited to lower acuity conditions, including infectious symptoms or preventive care. Finally, these results may not be generalizable to free-standing emergency rooms which are unaffiliated with a hospital and are staffed by board-certified emergency physicians who provide advanced cardiac life support and are able to transfer 
patients to a higher level of care. However, the painrelated diagnoses included in this study are commonly encountered in urgent care settings.

There were important study limitations. Our study was observational and cannot be interpreted as causal. We sought to minimize selection bias by including two urgent care clinics. To assess variability of patient type presenting to each clinic, we conducted a secondary analysis limiting our sample to the ED-based urgent care. Our results suggested there were some unobserved factors at the stand-alone urgent care that slightly increased the likelihood of opioid receipt at discharge. Next, our large cohort size led to statistical differences in some variables which may not be clinically significant. We created "in-clinic opioid" and "no in-clinic opioid" cohorts by determining if patients received clinically administered opioids during their index encounter. If a patient in the "no in-clinic opioid" group returned to the urgent care clinic following their index encounter and subsequently received an in-clinic opioid during the study period, this patient would remain in the "no inclinic opioid" group and would minimize the association between "in-clinic opioid" receipt and "progression to chronic opioid use." To quantify the potential effect this may have on our results, we identified subsequent healthcare encounters following the index encounter. This covariate was not included in our regression model because it did not reach statistical significance. We included patients without prescription opioid use at the time of the index encounter as inclusion criteria. We were unable to capture patients who filled an opioid prescription at non-affiliated pharmacies preceding their index encounter which may have caused misclassification bias for study inclusion. However, on average, $61 \%$ to $66 \%$ of medications prescribed by Denver Health clinicians are filled at a Denver Health-affiliated pharmacy. Similarly, we were unable to capture patients who filled opioid prescriptions outside of affiliated pharmacies in the 6 months following their index discharge. This could lead to under-ascertainment bias for the association between in-clinic opioid receipt and progression to chronic opioid use. We attempted to mitigate this bias by limiting our secondary outcome analysis to patients who were engaged in primary care and were more likely to fill their prescriptions at an affiliated pharmacy. Patients who received in-clinic opioids had slightly higher rates of 90-day ED and primary care visits. We were unable to ascertain the reason for the higher rates of utilization in these patients, whether it be an indication of greater severity of illness, or whether it was for ongoing pain control past the index encounter. Lastly, ICD coding practices may vary among clinicians leading to variability for study inclusion with painful conditions. Despite this limitation, the use of ICD codes remains a common method to study clinical outcomes.

\section{CONCLUSION}

We found a strong association between in-clinic opioid administration and discharge opioid prescriptions and progression to chronic opioid use in the urgent care setting. These results suggest that in-clinic opioid administration in urgent care could drive availability of opioids for diversion, and overdose, and may contribute to chronic opioid use. Future research may examine usual pain management strategies employed in urgent care settings across the USA.

Corresponding Author: Susan L. Calcaterra, MD, MPH; Division of General Internal Medicine, Department of Medicine, University of Colorado Aurora, CO, USA (e-mail: Susan.calcaterra@cuanschutz. edu).

\section{Compliance with Ethical Standards:}

Conflict of Interest: The authors declare that they do not have a conflict of interest.

\section{REFERENCES}

1. Deyo RA, Hallvik SE, Hildebran C, et al. Association Between Initial Opioid Prescribing Patterns and Subsequent Long-Term Use Among Opioid-Naïve Patients: A Statewide Retrospective Cohort Study. J Gen Intern Med 2017;32(1):21-27.

2. Butler MM, Ancona RM, Beauchamp GA, et al. Emergency Department Prescription Opioids as an Initial Exposure Preceding Addiction. Ann Emerg Med 2016;68(2):202-208.

3. Seth P, Rudd RA, Noonan RK, Haegerich TM. Quantifying the Epidemic of Prescription Opioid Overdose Deaths. Am J Public Health 2018;108(4):500-502.

4. LaPietra AM. Alternatives to Opioids for Acute Pain Management in the Emergency Department: Part II. Emerg Med Rep. 2016;37(20).

5. Duncan RW, Smith KL, Maguire M, Stader DE. Alternatives to opioids for pain management in the emergency department decreases opioid usage and maintains patient satisfaction. Am J Emerg Med 2019;37(1):38-44.

6. Healthcare A Convenient care: growth and staffing trends in urgent care and retail medicine. In:2016.

7. Wang $\mathbf{H}$, Robinson RD, Cowden CD, et al. Use of the SONET score to evaluate Urgent Care Center overcrowding: a prospective pilot study. BMJ Open 2015;5(4):e006860.

8. Weinick RM, Bristol SJ, DesRoches CM. Urgent care centers in the U.S.: findings from a national survey. BMC Health Serv Res. 2009;9:79.

9. Dowell D, Haegerich TM, Chou R. CDC guideline for prescribing opioids for chronic pain-United States, 2016. Jama 2016;315(15):1624-1645.

10. Herzig SJ, Mosher HJ, Calcaterra SL, Jena AB, Nuckols TK. Improving the safety of opioid use for acute non-cancer pain in hospitalized adults: a consensus statement from the Society of Hospital Medicine. J Hosp Med 2018;13(4):263.

11. Yeh BT. The controlled substances act: regulatory requirements. Congressional Research Service; 2012.

12. Administration USFaD. National Drug Code Directory. https://www.fda. gov/drugs/drug-approvals-and-databases/national-drug-code-directory. Updated 11/18/2019. Accessed 3 Dec 2019.

13. Vanderlip ER, Sullivan MD, Edlund MJ, et al. National study of discontinuation of long-term opioid therapy among veterans. Pain 2014;155(12):2673-2679.

14. Charlson ME, Pompei P, Ales KL, Mackenzie CR. A new method of classifying prognostic comorbidity in longitudinal studies: development and validation. J Chronic Dis 1987;40(5):373-383.

15. Calcaterra SL, Yamashita TE, Min SJ, Keniston A, Frank JW, Binswanger IA. Opioid Prescribing at Hospital Discharge Contributes to Chronic Opioid Use. J Gen Intern Med 2016;31(5):478-485. 
16. Weinick RM, Burns RM, Mehrotra A. Many emergency department visits could be managed at urgent care centers and retail clinics. Health Aff 2010;29(9):1630-1636.

17. Hsia RY, Friedman AB, Niedzwiecki M. Urgent care needs among nonurgent visits to the emergency department. JAMA Intern Med 2016;176(6):852-854.

18. Dart RC, Surratt HL, Cicero TJ, et al. Trends in opioid analgesic abuse and mortality in the United States. N Engl J Med 2015;372(3):241-248.

19. Warner M, Trinidad JP, Bastian BA, Minino AM, Hedegaard H. Drugs Most Frequently Involved in Drug Overdose Deaths: United States, 20102014. Natl Vital Stat Rep 2016;65(10):1-15.

20. Heitz C, Morgenstern J, Bond C, Milne WK. Hot Off the Press: A
Systematic Review And Meta-analysis of Ketamine as an Alternative to Opioids for Acute Pain in the Emergency Department. Acad Emerg Med Off J Soc Acad Emerg Med 2019;26(8):946-948.

21. Najjar M, Hall T, Estupinan B. Metoclopramide for Acute Migraine Treatment in the Emergency Department: An Effective Alternative to Opioids. Cureus 2017;9(4):e1181-e1181.

22. Todd KH. A Review of Current and Emerging Approaches to Pain Management in the Emergency Department. Pain Ther 2017;6(2):193202.

Publisher's Note: Springer Nature remains neutral with regard to jurisdictional claims in published maps and institutional affiliations. 